\title{
ASYMPTOTIC BOUNDARY VALUE PROBLEMS FOR EVOLUTION INCLUSIONS
}

TOMÁŠ FÜRST

Received 24 January 2005; Revised 12 July 2005; Accepted 17 July 2005

When solving boundary value problems on infinite intervals, it is possible to use continuation principles. Some of these principles take advantage of equipping the considered function spaces with topologies of uniform convergence on compact subintervals. This makes the representing solution operators compact (or condensing), but, on the other hand, spaces equipped with such topologies become more complicated. This paper shows interesting applications that use the strength of continuation principles and also presents a possible extension of such continuation principles to partial differential inclusions.

Copyright @ 2006 Tomáš Fürst. This is an open access article distributed under the Creative Commons Attribution License, which permits unrestricted use, distribution, and reproduction in any medium, provided the original work is properly cited.

\section{Introduction}

When solving boundary value problems on noncompact (in particular, on infinite) intervals, it is possible to use continuation principles. Unfortunatelly, one cannot simply extend the Leray-Schauder type theorems, because of the obstructions brought by the topology of uniform convergence on compact subintervals (see [1,2] or [6]). This topology makes the representing solution operators compact (or condensing) but, on the other hand, causes closed convex sets of certain type to have empty interiors. The main aim of this paper is to propose a modification of the continuation principle, originally given by Andres and Bader [1], to partial differential inclusions in Banach spaces and to present a nontrivial application of its usage.

Although the topology of uniform convergence on compact subintervals is wellknown (see [10]), for the sake of completeness we recall some of its interesting properties in Section 2. We show an example of a closed and convex set, which is often considered in applications and which has empty interior. We recall a way to overcome this drawback by considering relatively open subsets of closed convex sets (see $[2,6])$. Such sets are known to be absolute retracts (see [4]) and therefore are suitable for exploiting fixed point index techniques. We construct an example of such a relatively open set which is used in Section 3.

Hindawi Publishing Corporation

Boundary Value Problems

Volume 2006, Article ID 68329, Pages 1-12

DOI 10.1155/BVP/2006/68329 
Section 3 gives an example that fully uses the strength of the continuation principle proposed by Andres and Bader in [1]. We consider a first order differential inclusion with a special right-hand side and show the existence of an entirely bounded solution.

In the following section we propose a possible modification of the continuation principle, namely its application to boundary value problems for partial differential inclusions in Banach spaces. This section is a direct generalization of the results obtained by Andres and Bader in [1]. We show that the above mentioned continuation principles can be applied to a broad class of differential inclusions of parabolic type.

The last section gives an illustrating example of solving a boundary value problem for a particular differential inclusion of parabolic type. Inclusions of the considered type arise in solving nonlinear diffusion-type problems.

\section{Structure of Fréchet spaces}

When solving differential equations or inclusions on noncompact intervals, we often encounter the problem that the operators involved cease to be compact. To be able to exploit fixed point techniques even in this case, we take advantage of suitable topologies. This section deals with the topology of uniform convergence on compact subintervals in the space of continuous functions on the real axis. We collect here some of the known facts about the topology of uniform convergence on compact intervals.

Let $X$ be a Banach space. Consider space $\mathscr{C}(\mathbb{R}, X)$ of all $X$-valued functions continuous on the real axis. Let $\left\{K_{k}\right\}_{k \in \mathbb{N}}$ be a countable collection of compact intervals such that $K_{k} \subset K_{k+1}$ and $\mathbb{R}=\cup_{k \in \mathbb{N}} K_{k}$ and define collection of seminorms $\left\{p_{k}\right\}_{k \in \mathbb{N}}$ by

$$
p_{k}(f):=\max \left\{\|f(t)\|_{X} ; t \in K_{k}\right\} .
$$

Then space $\mathscr{C}(\mathbb{R}, X)$ equipped with the metric

$$
\mathbf{d}(f, g):=\sum_{j \in \mathbb{N}} \frac{1}{2^{j}} \frac{p_{j}(f-g)}{1+p_{j}(f-g)}
$$

becomes a complete metric space. (See [3, page 9].) It is a representant of the wide class of Fréchet spaces. Let us first mention some properties of sets in this topology.

First observe that any subset $A \subset \mathscr{C}(\mathbb{R}, X)$ is bounded, since for any pair $f, g \in \mathscr{C}(\mathbb{R}, X)$ it holds that

$$
\mathbf{d}(f, g)=\sum_{j \in \mathbb{N}} \frac{1}{2^{j}} \frac{p_{j}(f-g)}{1+p_{j}(f-g)} \leq \sum_{j \in \mathbb{N}} \frac{1}{2^{j}} \leq 1 .
$$

Any open ball $B(0, \varepsilon)$ in space $\mathscr{C}(\mathbb{R}, X)$ always contains functions which are unbounded in the usual sup-norm. Consider

$$
B(0, \varepsilon)=\{f \in \mathscr{C}(\mathbb{R}, X) ; \mathbf{d}(0, f)<\varepsilon\} .
$$

It is possible to find $n_{0} \in \mathbb{N}$ such that $\sum_{j=n_{0}}^{\infty}\left(1 / 2^{j}\right)<\varepsilon$. It follows that any continuous function $g$ which satisfies $g=0$ on $K_{n_{0}}$, satisfies estimate $\mathbf{d}(g, 0)<\varepsilon$ and therefore $g \in$ $B(0, \varepsilon)$. This shows that arbitrarily small balls contain functions that are unbounded in the 
usual sup-norm. Let us mention that since open sets in $\mathscr{C}(\mathbb{R}, X)$ are not even topologically bounded, space $\mathscr{C}(\mathbb{R}, X)$ is not normable.

Let $M>0$ and consider set

$$
Q:=\left\{q \in \mathscr{C}(\mathbb{R}, X) ;\|q(t)\|_{X} \leq M \forall t \in \mathbb{R}\right\},
$$

which often occurs in applications. It is easy to verify that $Q$ is closed and convex.

Observe also that $Q$ has an empty interior because any $q \in Q$ belongs to the boundary of $Q$.

Finally, we turn our attention to an example of a relatively open subset of $Q$. Such sets are important in applications since it is known that a relatively open subset of a closed, convex set in a Fréchet space is an absolute neighborhood retract. (See [4].) In Section 3 we present an interesting application of such sets.

Let $K \subset \mathbb{R}$ be compact and $O$ be a closed subset of $X$ which satisfies $O \subset B(0, M)$, where $M$ is given by (2.5). Consider the following obstacle set:

$$
Q^{\prime}:=\{q \in Q ; q(t) \notin O \forall t \in K\}
$$

Since $O$ "fits" into $Q$, it is evident that $Q$ ' is nonempty, $f \equiv M$ being one of its elements. It is easy to see that $Q^{\prime}$ is not open in $\mathscr{C}(\mathbb{R}, X)$, nevertheless, it can be shown that it is open in $Q$.

Let us conclude this section by the statement of a version of the classical Arzelà-Ascoli theorem.

Proposition 2.1. Let $X$ be a Banach space and $A$ an equicontinuous subset of $\mathscr{C}(\mathbb{R}, X)$ such that for all $t \in \mathbb{R}$, set $\{f(t) ; f \in A\}$ is precompact. Then $A$ is relatively compact.

\section{Continuation principle}

In this section we present an example of the usage of a continuation principle in Fréchet spaces. The continuation principle is based on the fixed point index for multivalued maps defined by Andres and Bader in [1]. For the sake of completness we collect here a simplified context of the index.

Let $F$ be a Fréchet space and $H: X \subset F \times[0,1] \multimap F$ a multivalued homotopy. We call a homotopy $H: X \times[0,1] \multimap F$ suitable if

(i) $X$ is a relatively open subset of a closed convex set in a Fréchet space $F$,

(ii) $H$ is upper semi-continuous with $R_{\delta}$ values,

(iii) $H$ is compact, (this condition can be replaced by the weaker requirement of $H$ being condensing, see [1])

(iv) for any fixed point $x \in H(x, t)$, there exist a neighborhood $u_{x}$ of $x$ in $X$ such that $H\left(u_{x} \times[0,1]\right) \subset X$.

We can now state the following lemma. (See [1, Corollary 12].) 
4 Asymptotic boundary value problems for evolution inclusions

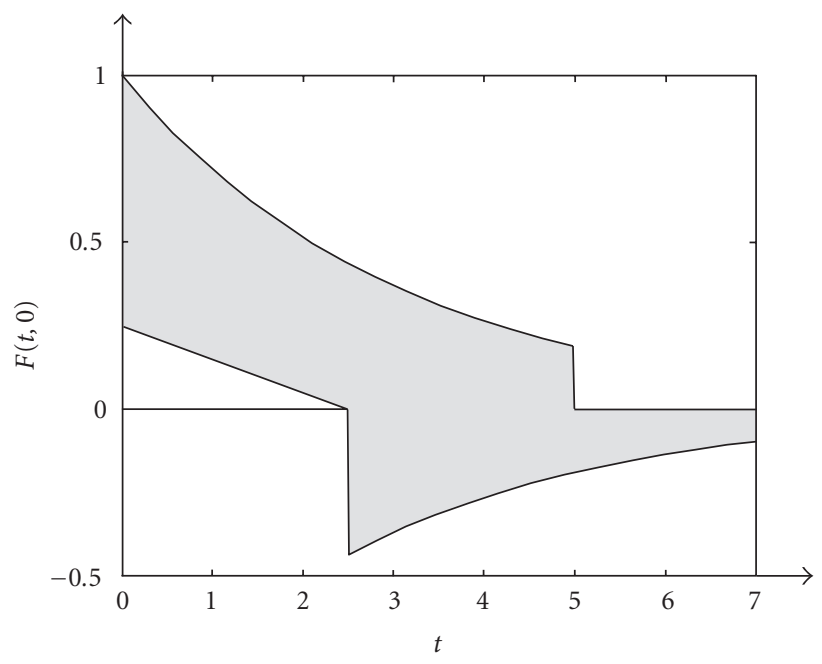

Figure 3.1. Graph of the multifunction $t \rightarrow F(t, 0)$.

Lemma 3.1. Let $H: X \times[0,1] \multimap F$ be a suitable homotopy such that $H(X, 0) \subset X$. Then $H(\cdot, 1)$ has a fixed point in $X$.

In applications, the strength of this principle is seldom used, we are thus going to present an example that fully uses the strength of the above lemma. Consider the inclusion

$$
u^{\prime}(t) \in F(t, u(t))
$$

for $t \in[0, \infty)$ with initial condition $u(0)=0$. We will look for a solution which satisfies inclusion (3.1) almost everywhere on $\mathbb{R}^{+}$in space $A C_{\text {loc }}\left(\mathbb{R}^{+}\right)$of all real valued functions locally absolutely continuous on $[0, \infty)$. This space is again endowed with the topology of uniform convergence on compact subintervals. In this particular case, we will look for bounded solutions which "go around" an obstacle $O:=[0,1 / 10]$ which is placed at $t=1$.

Let us consider particular right-hand side of inclusion (3.1):

$$
F(t, y):= \begin{cases}{\left[\frac{1}{4}-\frac{t}{10}, e^{-t / 3}+\frac{1}{3}\|y\|\right]} & \text { for } 0 \leq t \leq \frac{5}{2}, \\ {\left[-e^{-t / 3}-\frac{1}{3}\|y\|, e^{-t / 3}+\frac{1}{3}\|y\|\right]} & \text { for } \frac{5}{2} \leq t \leq 5 \\ {\left[-e^{-t / 3}-\frac{1}{3}\|y\|, 0\right]} & \text { for } 5 \leq t .\end{cases}
$$

Figure 3.1 shows the graph of the multifunction $t \rightarrow F(t, 0)$.

In order to prove the existence of an entirely bounded solution to (3.1) which goes around the obstacle, we again employ the linearization technique. We define the parameter set

$$
Q:=\left\{q \in A C_{\mathrm{loc}}\left(\mathbb{R}^{+}\right) ; q(0)=0,|q(t)| \leq 3 \forall t \in \mathbb{R}^{+}\right\}
$$


and the obstacle set

$$
Q^{\prime}:=\{q \in Q ; q(1) \notin O\}
$$

where $O=[0,1 / 10]$. With respect to the first section, we see that $Q$ is closed and convex subset of the Fréchet space $A C_{\mathrm{loc}}\left(\mathbb{R}^{+}\right)$and $Q^{\prime}$ is its relatively open subset. It is easy to verify, that the relative boundary $\partial_{Q} Q^{\prime}$ of $Q^{\prime}$ with respect to $Q$ is formed by those functions $q \in Q$ that satisfy $q(1)=0$ or $q(1)=1 / 10$.

Let us now define a homotopy $H:[0,1] \times Q^{\prime} \multimap A C_{\mathrm{loc}}\left(\mathbb{R}^{+}\right)$which to any $(\lambda, q) \in$ $[0,1] \times Q^{\prime}$ associates all solutions to

$$
u^{\prime}(t) \in F(t, \lambda q(t))
$$

where $F$ is given by (3.2) and boundary conditions of (3.1) remain valid. We want to apply Lemma 3.1 and show that $H(1, \cdot)$ has a fixed point in $Q^{\prime}$ which corresponds to the desired solution to the original problem. In order to draw such conclusion we need to show that

(1) $H$ is compact,

(2) $H$ is upper semi-continuous with compact and convex values,

(3) $H$ does not have any fixed points on the relative boundary $\partial_{Q} Q^{\prime}$,

(4) $H\left(0, Q^{\prime}\right) \subset Q^{\prime}$.

ad 1. We need to prove that $H\left([0,1] \times Q^{\prime}\right)$ is a relatively compact subset of $A C_{\text {loc }}\left(\mathbb{R}^{+}\right)$. In view of Proposition 2.1, it is sufficient to prove that $H\left([0,1] \times Q^{\prime}\right)$ is equicontinuous. The estimate $|q(t)| \leq 3$ for all $q \in Q^{\prime}$ and relation (3.2) imply that $|F(t, q(t))| \leq 2$ for all $t \in \mathbb{R}^{+}$. This means that $\left|u^{\prime}(t)\right| \leq 2$ for all $u \in H\left([0,1] \times Q^{\prime}\right)$. Such uniform boundedness of $u^{\prime}$ implies equicontinuity of $H\left([0,1] \times Q^{\prime}\right)$. This proves the compactness of homotopy $H$.

ad 2. Since $H$ is compact, it is sufficient to prove that $H$ has closed graph in order to conclude that it is upper semi-continuous. (See [3, Section I, Proposition 3.16].) Let us take a sequence $\left(\lambda_{n}, q_{n}, u_{n}\right)$ in the graph of $H$ which converges to $(\lambda, q, u)$ in $[0,1] \times Q^{\prime} \times$ $A C_{\text {loc }}\left(\mathbb{R}^{+}\right)$. We want to show that $u \in H(\lambda, q)$, which means that $u$ satisfies (3.5) for almost all $t \in \mathbb{R}^{+}$. Let us fix $t_{0} \in \mathbb{R}^{+}$such that $u^{\prime}{ }_{n}\left(t_{0}\right)$ and $u^{\prime}\left(t_{0}\right)$ exist. Note that the complement of the set of all such $t_{0} \in \mathbb{R}^{+}$has zero measure due to the local absolute continuity of $u_{n}$ and $u$. We know that

$$
u_{n}^{\prime}\left(t_{0}\right) \in F\left(t_{0}, \lambda_{n} q_{n}\left(t_{0}\right)\right)
$$

which together with the closedness of the values of $F$ implies

$$
u^{\prime}\left(t_{0}\right) \in \lim _{n \rightarrow \infty} F\left(t_{0}, \lambda_{n} q_{n}\left(t_{0}\right)\right) .
$$

The continuity of the mapping $y \rightarrow F\left(t_{0}, y\right)$, which is clear from (3.2), implies that

$$
\lim _{n \rightarrow \infty} F\left(t_{0}, \lambda_{n} q_{n}\left(t_{0}\right)\right)=F\left(t_{0}, \lambda q\left(t_{0}\right)\right)
$$


which completes the proof of the upper semi-continuity of $H$. Since $H$ is compact and the graph of $H$ is closed, we conclude that $H$ has compact values. The convexity of the values of $H$ follows from the convexity of the values of $F$. Note that compact and convex sets are, in particular, $R_{\delta}$ sets. (See [3, Chapter I.2].)

ad 3. Observe that $\partial_{Q} Q^{\prime}$ consists of such functions $q \in Q$ that satisfy $q(1)=0$ or $q(1)=$ $1 / 10$. Let us suppose there exists such $(\lambda, q) \in[0,1] \times Q^{\prime}$ that the solution $u$ of (3.5) satisfies $u(1)=1 / 10$. By the mean value theorem, this implies the existence of $t_{0} \in[0,1]$ such that $u^{\prime}\left(t_{0}\right) \leq 1 / 10$ which is a contradiction to the form of $F$ given by (3.2). Note that $1 / 4-t / 10>1 / 10$ for all $t \in[0,1]$. By the same argument we exclude the possibility that $u(1)=0$. This proves the nonexistence of fixed points on the relative boundary of $Q^{\prime}$.

ad 4. At last we show that $H\left(0, Q^{\prime}\right) \subset Q^{\prime}$. By an analogous argument to the previous paragraph, we can show that $u(1) \notin O$ for all $u \in H\left(0, Q^{\prime}\right)$. The particular form of $F$ implies that all $u \in H\left(0, Q^{\prime}\right)$ have to be nondecreasing on $[0,5 / 2]$ and nonincreasing on $[5, \infty)$. Simple calculation shows that $|u(t)| \leq 3$ for all $t \in \mathbb{R}^{+}$and $u \in H\left(0, Q^{\prime}\right)$ which completes the argument.

We have shown that all the assumption of Lemma 3.1 are satisfied and we can therefore establish the existence of a fixed point of $H(1, \cdot)$ which represents an entirely bounded solution to (3.1) which goes around the given obstacle.

\section{Application to partial differential inclusions}

In this section we present a possible extension of the continuation principle which shows the possibility of application of such principles in solving partial differential inclusions in Banach spaces.

Let us first introduce the Bochner space $W^{p, q}\left(K, V_{1}, V_{2}\right)$, where $K$ is a compact interval, $V_{1}$ and $V_{2}$ Banach spaces. The space $W^{p, q}\left(K, V_{1}, V_{2}\right)$ consists of functions $u$ such that

(i) $u(t) \in V_{1}$ for any $t \in K$,

(ii) $u \in L^{p}\left(K, V_{1}\right)$ in the sense that $\int_{K}\|u(t)\|_{V_{1}}^{p} d t$ is finite,

(iii) $(d u / d t)(t) \in V_{2}$ for any $t \in K$,

(iv) $d u / d t \in L^{q}\left(K, V_{2}\right)$ in the sense that $\int_{K}\|(d u / d t)(t)\|_{V_{2}}^{q} d t$ is finite,

where the derivatives are understood in the weak sense. If $K$ is a compact interval then $W^{p, q}\left(K, V_{1}, V_{2}\right)$, endowed with the norm

$$
\begin{aligned}
\|u\|_{W^{p, q}} & :=\|u\|_{L^{p}\left(K, V_{1}\right)}+\left\|\frac{d u}{d t}\right\|_{L^{q}\left(K, V_{2}\right)} \\
& =\left(\int_{K}\|u(t)\|_{V_{1}}^{p} d t\right)^{1 / p}+\left(\int_{K}\left\|\frac{d u}{d t}(t)\right\|_{V_{2}}^{q} d t\right)^{1 / q},
\end{aligned}
$$

becomes a Banach space.

If $I$ is an arbitrary interval, possibly whole $\mathbb{R}$, we let $K_{k}$ be a countable collection of compact intervals such that $K_{k} \subset K_{k+1}$ and $I \subset \cup_{k \in \mathrm{N}} K_{k}$ and for $v \in W^{p, q}\left(I, V_{1}, V_{2}\right)$ define 
the seminorm

$$
p_{k}(v):=\|v\|_{W^{p, q}\left(K_{k}, V_{1}, V_{2}\right)}
$$

Then the space $W^{p, q}\left(I, V_{1}, V_{2}\right)$ equipped with the metric

$$
\mathbf{d}\left(u_{1}, u_{2}\right):=\sum_{j \in \mathbf{N}} \frac{1}{2^{j}} \frac{p_{j}\left(u_{1}-u_{2}\right)}{1+p_{j}\left(u_{1}-u_{2}\right)}
$$

becomes a complete metric space.

Consider the inclusion

$$
\frac{d u}{d t}(t)+\mathbf{A} u(t) \in F(t, u(t))
$$

where $t \in I$ an arbitrary interval. Inclusion (4.4) has an abstract boundary condition

$$
u \in S \subset W^{p, q}\left(I, V_{1}, V_{2}\right)
$$

Let A : $V_{1} \rightarrow V_{2}$ be an operator (not necessarily linear), the properties of which are to be specified later. This operator represents the "spacial" part of the inclusion and in applications it usually stands for a differential operator of the second order from the Banach space $V$ into its dual $V^{*}$. Exponents $p$ and $q$ are usually dual and their qualification is given in Lemma 4.1. Let $F: I \times V_{1} \multimap V_{2}$ be a multivalued map and let $S$ be a nonempty subset of $W^{p, q}\left(I, V_{1}, V_{2}\right)$.

By a strong solution to problem (4.4) with boundary condition (4.5) we understand a function $u \in W^{p, q}\left(I, V_{1}, V_{2}\right)$ which satisfies boundary condition (4.5) and satisfies inclusion (4.4) on $V_{2}$ for almost all $t \in I$.

First, we are going to prove a technical lemma, which will be used later and which provides the qualification of the right-hand side of inclusion (4.4). We will need these definitions.

Let $F: I \times V_{1} \multimap V_{2}$ be a multivalued map. For $(t, v) \in I \times V_{1}$ we define

$$
\|F(t, v)\|_{V_{2}}:=\sup \left\{\|f\|_{V_{2}}: f \in F(t, v)\right\} .
$$

Let $F: I \times V_{1} \multimap V_{2}$ be a multivalued map. We define the Nemyckii operator $N_{F}: L^{p}\left(I, V_{1}\right)$ $\multimap L^{q}\left(I, V_{2}\right)$ by

$$
N_{F}(v):=\left\{f \in L^{q}\left(I, V_{2}\right): f(t) \in F(t, v(t)) \text { for almost all } t \in I\right\} .
$$

The following lemma justifies the above definition and describes the properties of the Nemyckii operator.

Lemma 4.1. Let $K$ be a compact interval, $p, q \geq 1, V_{1} \hookrightarrow V_{2}$ continuously and let $F: K \times$ $V_{1} \multimap V_{2}$ satisfy conditions

(i) $F(t, v) \subset V_{2}$ is nonempty, closed and convex for all $(t, v) \in K \times V_{1}$,

(ii) $F(t, \cdot): V_{1} \multimap V_{2}$ is upper semi-continuous for almost all $t \in K$, 
8 Asymptotic boundary value problems for evolution inclusions

(iii) $F(t, v): K \times V_{1} \multimap V_{2}$ is product measurable,

(iv) $\|F(t, v)\|_{V_{2}} \leq \alpha(t)+\beta\|v\|_{V_{1}}^{p / q}$, where $\alpha \in L^{q}(K)$ and $\beta \geq 0$,

then the Nemyckii operator $N_{F}: L^{p}\left(K, V_{1}\right) \multimap L^{q}\left(K, V_{2}\right)$ has nonempty, closed and convex values and is upper semi-continuous. Moreover, it satisfies the following property:

$$
\left.\begin{array}{l}
u_{n} \longrightarrow u \text { in } W^{p, q}\left(I, V_{1}, V_{2}\right) \\
f_{n} \in N_{F}\left(u_{n}\right), f_{n} \longrightarrow f \text { weakly in } L^{q}\left(K, V_{2}\right)
\end{array}\right\} \text { implies that } f \in N_{F}(u) .
$$

Proof. The proof of the first part of the statement follows directly from [8, page 237]. For $p, q \geq 1$ and $V_{1} \hookrightarrow V_{2}$ it holds that $W^{p, q}\left(K, V_{1}, V_{2}\right) \hookrightarrow C\left(K, V_{2}\right)$. (See [7, page 173].) The proof of part two now follows from [1] (see [1, Lemma 14]) and from the obvious embedding $L^{q}(K) \hookrightarrow L^{1}(K)$, for $q \geq 1$.

We now specify the properties of the operator A. Let A be locally bounded in the sense that

$$
\|\mathbf{A} v\|_{V_{2}} \leq C\left(1+\|v\|_{V_{1}}^{p / q}\right)
$$

for any $v \in V_{1}$. Observe that for $u \in L^{p}\left(K, V_{1}\right)$ we then have

$$
\int_{K}\|\mathbf{A} u(t)\|_{V_{2}}^{q} d t \leq \int_{K} C^{q}\left(1+\|u(t)\|_{V_{1}}^{p / q}\right)^{q} \leq M \int_{K}\|u(t)\|_{V_{1}}^{p},
$$

which is finite because $u \in L^{p}\left(K, V_{1}\right)$. We further define operator $\mathscr{A}$ by

$$
(\mathscr{A} u)(t):=\mathbf{A} u(t)
$$

If $\mathbf{A}$ is bounded in the sense of (4.9), then the above calculation shows that $\mathscr{A}: L^{p}(K$, $\left.V_{1}\right) \rightarrow L^{q}\left(K, V_{2}\right)$.

We will further need some notion of continuity of $\mathscr{A}$. The following definition turns out to be optimal.

Operator $\mathscr{A}: L^{p}\left(I, V_{1}\right) \rightarrow L^{q}\left(I, V_{2}\right)$ is demicontinuous if $u_{n} \rightarrow u$ in $L^{p}\left(I, V_{1}\right)$ implies $\mathscr{A}\left(u_{n}\right) \rightarrow \mathscr{A}(u)$ weakly in $L^{q}\left(I, V_{2}\right)$. Operator $\mathscr{A}$ is locally demicontinuous if it is demicontinuous for any $K \subset I$ compact.

We now want to employ the standard technique of partial linearization of the righthand side of inclusion (4.4). We need to prove that the solution operator of the linearized problem has closed graph. This, together with some compactness argument, will guarantee the upper semi-continuity of the solution operator.

Lemma 4.2. Let $G: I \times V_{1} \times V_{1} \multimap V_{2}$ satisfy assumptions (i)-(iv) of Lemma 4.1 jointly in $V_{1} \times V_{1}$. Let $S$ be a nonempty and closed subset of $W^{p, q}\left(I, V_{1}, V_{2}\right)$. Let $\mathbf{A}$ be bounded in the sense of (4.9) and let $\mathscr{A}$ defined in (4.11) be locally demicontinuous. Let there exist a closed $Q \subset W^{p, q}\left(I, V_{1}, V_{2}\right)$ such that for any $q \in Q$ the problem

$$
\frac{d u}{d t}(t)+\mathbf{A} u(t) \in G(t, u(t), q(t)), \quad u \in S
$$

has a solution. Denote by $T: Q \multimap S$ the solution mapping. Then $T$ has closed graph. 
Proof. Choose $\left(q_{n}, u_{n}\right)$ an arbitrary sequence in the graph of $T$ such that $\left(q_{n}, u_{n}\right) \rightarrow\left(q_{0}, u_{0}\right)$ in $W^{p, q}\left(I, V_{1}, V_{2}\right) \times W^{p, q}\left(I, V_{1}, V_{2}\right)$. Since $S$ is closed, we have $u_{0} \in S$. We need to show that $u_{0} \in T\left(q_{0}\right)$ which means that

$$
\frac{d u_{0}}{d t}(t)+\mathbf{A} u_{0}(t) \in G\left(t, u_{0}(t), q_{0}(t)\right)
$$

for almost all $t \in I$. We know that

$$
\frac{d u_{n}}{d t}(t)+\mathbf{A} u_{n}(t) \in G\left(t, u_{n}(t), q_{n}(t)\right)
$$

Let us confine to any $K \subset I$ compact. In view of Lemma 4.1, it is sufficient to show that

$$
\frac{d u_{n}}{d t}+\mathscr{A}\left(u_{n}\right) \longrightarrow \frac{d u_{0}}{d t}+\mathscr{A}\left(u_{0}\right) \quad \text { weakly in } L^{q}\left(K, V_{2}\right)
$$

The convergence $d u_{n} / d t \rightarrow d u_{0} / d t$ in $L^{q}\left(K, V_{2}\right)$ is ensured by the first step of the proof and the weak convergence $\mathscr{A}\left(u_{n}\right) \rightarrow \mathscr{A}\left(u_{0}\right)$ follows from the demicontinuity of $\mathscr{A}$. This proves (4.15) and in view of Lemma 4.1 indeed (4.13) holds for almost all $t \in K$. Since $K$ is arbitrary, we conclude that (4.13) holds for almost all $t \in I$, which means $u_{0} \in T\left(q_{0}\right)$.

We are now in the position to prove the main result of this section-the continuation principle. We again consider problem (4.4) with boundary condition (4.5).

Proposition 4.3. Let $G: I \times V_{1} \times V_{1} \times[0,1] \multimap V_{2}$ satisfy the assumptions of Lemma 4.1 jointly in $V_{1} \times V_{1}$ and uniformly on $[0,1]$. Let $G(t, c, c, 1) \subset F(t, c)$ for all $(t, c) \in I \times V_{1}$. Let A be locally bounded in the sense of (4.9) and A locally demicontinuous. Let $S \subset W^{p, q}\left(I, V_{1}\right.$, $\left.V_{2}\right)$ be nonempty and closed. Let there exist a closed convex $Q \subset W^{p, q}\left(I, V_{1}, V_{2}\right)$ such that for any $(s, q) \in[0,1] \times Q$ the problem

$$
\frac{d u}{d t}(t)+\mathbf{A} u(t) \in G(t, u(t), q(t), s), \quad u \in S,
$$

has a solution such that the solution operator $T:[0,1] \times Q \multimap S$ has the following properties:

(i) for any $(s, q) \in[0,1] \times Q$, set $T(s, q)$ is $R_{\delta}$,

(ii) $T$ is compact,

(iii) $T(Q, 0) \subset Q$,

(iv) for any fixed point $q \in T(s, q)$, there exists a neighborhood $U_{q}$ in $Q$ such that $T([0$, 1] $\left.\times u_{q}\right) \subset Q$.

Then problem (4.4) together with boundary condition (4.5) has a solution.

Proof. In view of Lemma 4.2 we conclude that the solution operator $T$ has closed graph. This, together with assumption (ii) gives the upper semi-continuity of $T$. (See again [3, Section I, Proposition 3.16].) Assumptions (i), (ii), and (iv) ensure that $T$ is a suitable homotopy in the sense of the previous section. We can therefore apply Lemma 3.1, the assumption of which is guaranteed by (iii), and conclude that $T(u, 1)$ has a fixed point 
$u \in T(u, 1)$ which is a solution to the inclusion

$$
\frac{d u}{d t}(t)+\mathbf{A} u(t) \in G(t, u(t), u(t), 1), \quad u \in S .
$$

Relation $G(t, c, c, 1) \subset F(t, c)$ ensures that this fixed point $u$ is a solution to the original problem.

\section{Illustrating example}

As an example we will consider inclusion

$$
\frac{d u}{d t}-\Delta u \in F(t, u), \quad u \in S
$$

where $t \in I$ an arbitrary interval and $x \in \Omega$ a bounded subset of $\mathbb{R}^{n}$ and the properties of $F$ and $S$ are to be specified later. We will look for a strong solution to problem (5.1) in space $W^{2,2}\left(I, W^{1,2}(\Omega), L^{2}(\Omega)\right)$. For the sake of simplicity, we denote

$$
\begin{aligned}
\mathbf{W}^{2,2} & :=W^{2,2}\left(I, W^{1,2}(\Omega), L^{2}(\Omega)\right), \\
\mathbf{L}_{\mathbf{L}}^{2} & :=L^{2}\left(I, L^{2}(\Omega)\right) .
\end{aligned}
$$

We will now specify $S$ as follows:

$$
S:=\left\{u \in \mathbf{W}^{2,2}: u(0)=u_{0}, u=0 \forall(t, x) \in I \times \partial \Omega\right\} .
$$

Note that this definition has meaning, because the functions involved have continuous representants.

Let the right-hand side $F: I \times L^{2}(\Omega) \multimap L^{2}(\Omega)$ satisfy the following assumptions:

(i) $F(t, v)$ is nonempty, closed and convex for all $(t, v) \in I \times L^{2}(\Omega)$,

(ii) $F$ is product measurable,

(iii) $F(t, \cdot)$ is upper semi-continuous,

(iv) $|F(t, v)| \leq \alpha(t)+\beta\|v\|_{L^{2}}$, where $\alpha \in L^{2}(I)$ and $\beta<1 / 2$.

It follows from Lemma 4.1 , that $N_{F}: \mathrm{L}_{\mathbf{L}}^{2} \multimap \mathrm{L}_{\mathbf{L}}^{2}$ is upper semi-continuous with nonempty, closed and convex values.

Let us now define the linearization set

$$
Q:=\left\{q \in \mathbf{W}^{2,2}:\|q(t)\|_{L^{2}} \leq M \forall t \in I\right\},
$$

where $M$ is to be specified later. Take $q \in Q$ arbitrary. The obvious embedding $\mathbf{W}^{2,2} \hookrightarrow \mathbf{L}_{\mathbf{L}}^{2}$ gives a nonempty, closed and convex $N_{F}(q) \subset \mathbf{L}_{\mathbf{L}}^{2}$. For arbitrary $f \in N_{F}(q)$ we will solve the linearized problem

$$
\frac{d u}{d t}-\Delta u=f(t), \quad u \in S
$$

It is well-known that problem (5.5) has a unique strong solution $u \in \mathbf{W}^{2,2}$. (See [5, Chapter 7, Theorem 5].) Moreover, the solution operator $K: \mathbf{L}_{\mathbf{L}}^{2} \rightarrow \mathbf{W}^{2,2}$ such that $K f=u$ is linear and continuous. Let us now denote $u$ the solution of (5.5) for $f \in N_{F}(q)$. We will 
first prove that $u \in Q$. We need to show that $\|u(t)\|_{L^{2}} \leq M$. Multiplying (5.5) by $u$, integrating over $\Omega$ and integrating from 0 to $t$, we obtain

$$
\int_{0}^{t} \int_{\Omega} \frac{d u}{d t} u-\int_{0}^{t} \int_{\Omega}(\Delta u) u=\int_{0}^{t} \int_{\Omega} f u .
$$

Applying the Green theorem and rearranging the terms we obtain

$$
\int_{0}^{t} \frac{1}{2} \frac{d}{d t}\|u\|_{L^{2}}^{2}-\int_{0}^{t} \int_{\partial \Omega} \frac{\partial u}{\partial \nu} u+\int_{0}^{t} \int_{\Omega} \nabla u \cdot \nabla u=\int_{0}^{t} \int_{\Omega} f u
$$

Since $u \in S$, the boundary integral equals to zero. We apply the Hölder and Young inequalities to the right hand-side and obtain

$$
\frac{1}{2}\|u(t)\|_{L^{2}}^{2}-\frac{1}{2}\left\|u_{0}\right\|_{L^{2}}^{2}+\int_{0}^{t}\|\nabla u\|_{L^{2}}^{2} \leq C_{1} \int_{0}^{t}\|f\|_{L^{2}}^{2}+\frac{1}{2} \int_{0}^{t}\|u\|_{L^{2}}^{2}
$$

such that $C_{1}<2$. Since $u=0$ on $\partial \Omega$, we employ the Poincaré inequality to obtain

$$
\frac{1}{2}\|u(t)\|_{L^{2}}^{2}+B \int_{0}^{t}\|u\|_{W^{1,2}}^{2} \leq C_{1} \int_{0}^{t}\|f\|_{L^{2}}^{2}+\frac{1}{2}\left\|u_{0}\right\|_{L^{2}}^{2}
$$

Using the properties of $F$ and the definition of $Q$, we obtain

$$
\int_{0}^{t}\|f\|_{L^{2}}^{2} \leq \int_{0}^{t} \alpha^{2}+\beta^{2} M \leq C_{2}+\beta^{2} M
$$

which substituted back to (5.9) gives

$$
\frac{1}{2}\|u(t)\|_{L^{2}}^{2} \leq C_{3}+C_{1} \beta^{2} M
$$

Since $C_{3}>0, C_{1}<2, \beta^{2}<1 / 4$, it is possible to set $M$, such that

$$
C_{3}+C_{1} \beta^{2} M \leq \frac{M}{2} .
$$

Indeed, it is sufficient to take

$$
M:=\frac{2 C_{3}}{1-2 C_{1} \beta^{2}}
$$

This shows that any solution $u$ to problem (5.5) satisfies $u \in S$.

Altogether, we have the following sequence of mappings:

$$
q \in Q \subset \mathbf{W}^{2,2} \longrightarrow q \in \mathbf{L}_{\mathbf{L}}^{2} \multimap N_{F}(q) \subset \mathbf{L}_{\mathbf{L}}^{2} \longrightarrow K\left(N_{F}(q)\right) \subset Q \subset \mathbf{W}^{2,2},
$$

where the first mapping is the compact inclusion $\mathbf{W}^{2,2} \hookrightarrow \mathrm{L}_{\mathrm{L}}^{2}$, the second one is the Nemyckii map with closed and convex values, and the third one is the linear and continuous solution map of the linearized problem. The composition $T: Q \rightarrow Q$ of these three maps is therefore an upper semi-continous map with closed and convex values. To conclude 
that $T$ has a fixed point, we need to ensure that $T$ is compact or, at least, condensing with respect to a suitable measure of noncompactness. Due to the compactness of the first inclusion, it is sufficient that $N_{F}$ maps compact sets on compact (or at least precompact) sets. Let us mention that this condition is satisfied for example, if $F$ is single valued (then the single valued Nemyckii map becomes continuous), or if $N_{F}(q)$ consists of finite number of points (compare [9]).

\section{Acknowledgment}

The author would like to thank Professor Jan Andres for bringing up the topic, for fruitful discussions on the theme, and for numerous inspirating consultations.

\section{References}

[1] J. Andres and R. Bader, Asymptotic boundary value problems in Banach spaces, Journal of Mathematical Analysis and Applications 274 (2002), no. 1, 437-457.

[2] J. Andres, G. Gabor, and L. Górniewicz, Boundary value problems on infinite intervals, Transactions of the American Mathematical Society 351 (1999), no. 12, 4861-4903.

[3] J. Andres and L. Górniewicz, Topological Fixed Point Principles for Boundary Value Problems, Topological Fixed Point Theory and Its Applications, vol. 1, Kluwer Academic, Dordrecht, 2003.

[4] K. Borsuk, Theory of Retracts, Monografie Matematyczne, vol. 44, Państwowe Wydawnictwo Naukowe, Warsaw, 1967.

[5] L. C. Evans, Partial Differential Equations, Graduate Studies in Mathematics, vol. 19, American Mathematical Society, Rhode Island, 1998.

[6] M. Furi and M. P. Pera, On the fixed point index in locally convex spaces, Proceedings of the Royal Society of Edinburgh. Section A. Mathematics 106 (1987), no. 1-2, 161-168.

[7] H. Gajewski, K. Gröger, and K. Zacharias, Nichtlineare Operatorgleichungen und Operatordifferentialgleichungen, Mathematische Lehrbücher und Monographien, II. Abteilung, Mathematische Monographien, vol. 38, Akademie, Berlin, 1974.

[8] S. Hu and N. S. Papageorgiou, Handbook of Multivalued Analysis. Vol. I. Theory, Mathematics and Its Applications, vol. 419, Kluwer Academic, Dordrecht, 1997.

[9] A. Margheri and P. Zecca, Solution sets and boundary value problems in Banach spaces, Topological Methods in Nonlinear Analysis 2 (1993), no. 1, 179-188.

[10] H. H. Schaefer, Topological Vector Spaces, The Macmillan, New York; Collier-Macmillan, London, 1966.

Tomáš Fürst: Department of Mathematical Analysis and Applications of Mathematics, Faculty of Science, Palacký University, Tomkova 40, 77900 Olomouc, Czech Republic

E-mail address: tomas.furst@seznam.cz 